\title{
2D molybdenum disulphide nanosheets incorporated with single heteroatoms for electrochemical hydrogen evolution reaction
}

Received 00th January 20xx, Accepted 00th January 20xx

DOI: $10.1039 / x 0 x \times 00000 x$

\author{
Thomas H.M. Lau ${ }^{\mathrm{a}}$, John S. Foord ${ }^{\mathrm{a}}$, S.C. Edman Tsang ${ }^{\mathrm{a}^{*}}$
}

\begin{abstract}
2D nanosheets give enhanced surface area to volume ratios in particle morphology and they can also provide defined surface sites to disperse foregin atoms. Placing atoms of catalytic interests on the 2D nanosheets as Single Atom Catalysts (SAC) represents one of the novel approaches due to their unique but tunable electronic and steric characterstics. Here in this mini-review, we particularly highlight some recent and important developments on heteroatoms doped MoS $_{2}$ nanosheets (SAC-MoS $\mathrm{S}_{2}$ ) as catalysts for electrochemical hydrogen evolution reaction (HER) from water, which may form the core to open up to a flagship of important renewable tehnologies in future. It is shown that the nature of dopants, doping positions and polytypes of $\mathrm{MoS}_{2}$ nanosheets are the determing factors on the overall catalytic abilities of these functionalised nanosheets. This may serve to obtain atomic models which lead to further understanding on 'metal-support interaction' in catalysis.
\end{abstract}

\section{Introduction}

The subtle climate change due to excessive carbon emissions to atmosphere has prompted our community to switch energy sources from oil, gas and coal to renewables such as solar, wind power and tidal wave, etc. Green $\mathrm{H}_{2}$ manufacture from electrolysis of water over efficient electrocatalyst is the core technology to harness these renewables. The produced $\mathrm{H}_{2}$ with zero carbon emission can be directly used in combustion engines, fuel cells, or for reduction of $\mathrm{CO}_{2}$, etc. Alternatively, it can be stored as chemicals such as ammonia or methanol to facilitate transport and uses in other regions. Although noble metal catalysts give satisfactory performance in electrolyser, their high cost and scarcity have compelled chemical manufacturers to find greener and more cost-effective alternatives. Thanks to the advances of nanotechnology, twodimensional (2D) metallic or semi-conductive materials such as graphite based nanostructures ${ }^{1,2}$, transition metal dichalcogenides (TMDC) ${ }^{3-5}$, transition metal oxides $(\mathrm{TMO})^{6,7}$, and transition metal nitrides (TMN $)^{8,9}$, have been thoroughly studied and characterised. These atomically thick 2D nanosheets possess a much higher surface-to-volume-ratio than three-dimensional (3D) nanomaterials. Since more active sites located at the surface can be accessed for the desired reactions, they generally exhibit better catalytic performances than their 3D counterparts. However, they are still incomparable to conventional noble metal catalysts such as $\mathrm{Pt}$ and Ru. Thus, further modifications have been attempted to improve the material properties of these 2D catalysts. The general idea is to either increase the number of active sites available or enhance the intrinsic activity of each active site. For example, the former approach can be achieved by exposing more edge sites to by introducing surface defects ${ }^{10-14}$ while the latter one can involve changing the electronic structure of the responsible atoms at the active sites via strain eningeering ${ }^{15-17}$.
One of the newest modification methods to functionalise 2D nanosheets is to incorporate single heteroatoms onto the large basal surface of the 2D catalysts. Previously, single-atom catalysts (SAC) generally referred to supporting materials confined with isolated single heteroatoms, which the dopants became the new active sites and contribute their catalytic activities in the reactions of interest. ${ }^{18-20}$ Since the dispersed atomic dopants maximise metal utilisation, which makes the catalyst excelled in small molecules activation. Such combination of SAC and 2D catalysts creates a new group of materials, which is multifunctional in catalysis due to the coexistence of two types of active sites on the surface, potentially making more complicated reaction mechanisms plausible. While this new type of catalysts generates vast interests as it combines the knowledge of the two hottest research areas in catalysis, the research field has yet been well explored. Several excellent review articles have been published to highlight some recent research works in the area of SAC. ${ }^{21-23}$ Similarly, there are some good reviews focusing on using 2-D materials such as graphene, molybdenum disulphide ( $\left.\mathrm{MoS}_{2}\right)$, and graphitic carbon nitride $\left(\mathrm{g}-\mathrm{C}_{3} \mathrm{~N}_{4}\right)$ etc as catalysts in electrochemical reactions including HER when doped with heteroatoms. The challenges of synthesising and analysing SAC2D catalysts have also been reviewed therein. The readers should refer to these articles for reference without being repeatedly discussed in this article. In attempt to provide insights for future research in this field of SAC and 2D materials in combine, this mini-review will particularly examine some selected key publications on single atoms doped $\mathrm{MoS}_{2}$ nanosheets $\left(\mathrm{SAC}-\mathrm{MoS}_{2}\right.$ ) in HER as a short but sharp focused review. The synthetic approaches and the electrocatalytic activities of these materials will be thoroughly analysed with the aim to better understand the structure-activity relationship of SAC-MoS

\section{Polytypes of 2D MoS2}


Table 1. Highlighted publications showing synthetic methods, polytypes of 2D $\mathrm{MoS}_{2}$ nanosheets, metal doping concentration and their preferable doping position(s).

\begin{tabular}{|c|c|c|c|c|c|}
\hline Entry & Catalyst & Synthetic Method & Polytype & $\begin{array}{l}\text { Doping Concentration and Preferable Doping } \\
\text { Position }\end{array}$ & Ref. \\
\hline $1 \mathrm{~A}$ & $\mathrm{MoS}_{2}$ & Mechanical exfoliation under sonication & $2 \mathrm{H}$ & -- & 25 \\
\hline 1B & $\mathrm{MoS}_{2}$ & Lithium intercalation & $1 \mathrm{~T}$ & -- & 25 \\
\hline $2 A$ & $\mathrm{Co}-\mathrm{MoS}_{2}$ & $\begin{array}{l}\text { One pot solvothermal reaction } \\
\text { at } 400^{\circ} \mathrm{C}, 4 \mathrm{hrs}\end{array}$ & $2 \mathrm{H}$ & $1.7 \%$ Co, Mo substitution sites at edge sites & 26 \\
\hline 2B & Pt-MoS 2 & $\begin{array}{l}\text { One pot solvothermal reaction } \\
\qquad \text { at } 400^{\circ} \mathrm{C}, 4 \mathrm{hrs}\end{array}$ & $2 \mathrm{H}$ & $1.7 \% \mathrm{Pt}, \mathrm{Mo}$ substitution sites at edge sites & 26 \\
\hline 3 & $\begin{array}{l}\text { Co-MoS } \\
\text { with S defects }\end{array}$ & $\begin{array}{l}\text { Lithium intercalation, } \\
\text { hydrothermal doping at } 180^{\circ} \mathrm{C}, 12 \mathrm{hrs}\end{array}$ & $1 \mathrm{H}$ & $\begin{array}{l}3 \% \text { Co, Mo atop sites } \\
\text { at basal plane }\end{array}$ & 27 \\
\hline 4 & $\mathrm{Pd}-\mathrm{MoS}_{2}$ & $\begin{array}{l}\text { One pot solvothermal reaction } \\
\text { at } 180^{\circ} \mathrm{C}, 24 \mathrm{hrs}\end{array}$ & $1 \mathrm{~T}$ & 1\% Pd, Mo substitution sites at basal plane & 28 \\
\hline $5 A$ & $\mathrm{Pt}-\mathrm{MoS}_{2}$ & $\begin{array}{l}\text { Lithium intercalation, } \\
\text { sonochemical doping at room temperature, } 24 \mathrm{hrs}\end{array}$ & $1 T$ & $\begin{array}{l}\text { 3\% Pt, Mo atop sites } \\
\text { at basal plane }\end{array}$ & 29 \\
\hline $5 B$ & $\mathrm{Pd}-\mathrm{MoS}_{2}$ & $\begin{array}{l}\text { Lithium intercalation, } \\
\text { sonochemical doping at room temperature, } 24 \mathrm{hrs}\end{array}$ & $1 T$ & $\begin{array}{l}3 \% \text { Pd, Mo atop sites } \\
\text { at basal plane }\end{array}$ & 29 \\
\hline 6 & $\mathrm{P}-\mathrm{MoS}_{2}$ & $\begin{array}{l}\text { One pot solvothermal reaction } \\
\text { at } 200^{\circ} \mathrm{C}, 24 \mathrm{hrs}\end{array}$ & $1 T$ & $5 \% \mathrm{P}, \mathrm{S}$ substitution at basal plane & 30 \\
\hline 7 & $\mathrm{~N}-\mathrm{MoS}_{2}$ & $\begin{array}{l}\text { One pot solvothermal reaction } \\
\text { at } 200^{\circ} \mathrm{C}, 24 \mathrm{hrs}\end{array}$ & $1 \mathrm{~T}$ & $5.7 \% \mathrm{~N}$, Mo substitution sites at basal plane & 31 \\
\hline
\end{tabular}

$\mathrm{MoS}_{2}$ is a layered structure with each triatomic monolayer consisting of a hexagonal plane of molybdenum atoms sandwiched between two other hexagonal planes of sulphur atoms via strong in-plane covalent bonding. Adjacent layers are stacked together by weak out-of-plane van der Waals interactions. ${ }^{24}$ There are multiple polytypes of $\mathrm{MoS}_{2}: 2 \mathrm{H}-\mathrm{MoS}_{2}$, $3 \mathrm{R}-\mathrm{MoS}_{2}$, and $1 \mathrm{~T}-\mathrm{MoS}_{2}$, which they give distinctive electronic properties. The digit refers to number of layers in the unit cell while the letter " $H$ ", " $R$ ", and " $T$ " indicate hexagonal ( $D_{3 h}$ group), rhombohedral ( $C_{3 v}^{5}$ group), and tetragonal ( $D_{3 d}$ group) symmetry respectively. Both $2 \mathrm{H}-\mathrm{MoS}_{2}$ and $3 \mathrm{R}-\mathrm{MoS}_{2}$ are semiconducting while the $1 \mathrm{~T}-\mathrm{MoS}_{2}$ displays a metallic behaviour. 1T$\mathrm{MoS}_{2}$ is found to possess an electrical conductivity of around $10^{7}$ times higher than that of $2 \mathrm{H}-\mathrm{MoS}_{2}{ }^{32}$ If $2 \mathrm{H}$ - and $3 \mathrm{R}-\mathrm{MoS}_{2}$ crystals are exfoliated into monolayers, the polytype will then be referred as $1 \mathrm{H}$ to denote a monolayer structure. $1 \mathrm{~T}-\mathrm{MoS}_{2}$ is metastable as its unit cell is the distorted form of $2 \mathrm{H}-\mathrm{MoS}_{2}$. Thus, it needs to be stabilised or else it will be converted back to $2 \mathrm{H}-\mathrm{MoS}_{2}$ upon heating above $95^{\circ} \mathrm{C} .{ }^{33}$ For example, computational calculations suggested that the $1 \mathrm{~T}$ polytype can be distorted into a lower energy $1 T^{\prime}$ structure due to the stabilisation of hydrogen adsorption in HER. ${ }^{34,35}$

The choice of the $\mathrm{MoS}_{2}$ polytype not only affects the overall electrical conductivity but also the density state of the active sites. During catalysis, the Mo edges (1010) and the $S$ edges $(1 \overline{0} 10)$ of $\mathrm{MoS}_{2}$ can accept and donate electrons like a metal surface. ${ }^{36}$ The contribution by the edge sites however is far less important for 2D MoS 2 due to the presence of larger basal surface. The basal plane (0001) of semiconducting $\mathrm{MoS}_{2}$ has been verified to be chemically inert both experimentally ${ }^{15}$ and computationally ${ }^{37}$ as further modification is required to activate it. In contrary, the metallic feature of $1 \mathrm{~T}-\mathrm{MoS}_{2}$ has made the basal sites highly active for electrocatalysis. ${ }^{38,39}$ Top-down and bottom-up methods are two major approaches to obtain 2D $\mathrm{MoS}_{2}$ nanosheets. The former method is to isolate monolayers from bulk $\mathrm{MoS}_{2}$ crystals by weakening the interlayer van der Waals interactions. Lithium intercalation is one example to create monolayer $1 \mathrm{~T}-\mathrm{MoS}_{2}$ with rich S-defects. ${ }^{40-42}$ The latter method involves reacting Mo precursors with $\mathrm{S}$ sources to grow $\mathrm{MoS}_{2}$ unit by unit. Chemical vapour deposition (CVD) ${ }^{43-46}$ and one-pot hydrothermal/solvothermal synthesis ${ }^{47-49}$ are often used to grow high-quality ultrathin $2 \mathrm{H}-\mathrm{MoS}_{2}$ or monolayer $1 \mathrm{H}$ $\mathrm{MoS}_{2}$.

\section{Synthetic Methods of SAC-MoS2}

Table 1 lists the synthetic approaches of selected publications that were adopted in preparing different SACs$\mathrm{MoS}_{2}$ for electrochemical HER. It has shown that the synthesis of SAC-MoS ${ }_{2}$ nanosheets is predominantly achieved by two different synthetic routes: (1) incorporating single heteroatoms onto the as-prepared 2D MoS 2 nanosheets with the desired polytypes and (2) directly growing the catalysts through hydrothermal/ solvothermal reaction. The synthetic route will largely influence the doping position of the heteroatoms adopted in the SAC-MoS 2 catalysts. It was revealed that there are four general doping sites for single molecular layer $\mathrm{MoS}_{2}$, namely Mo atop site, $\mathrm{S}$ substitution site, Mo substitution site, and the hollow site. ${ }^{50}$ For the first route, $\mathrm{MoS}_{2}$ nanosheets are first synthesised beforehand usually by lithium intercalation (Method 1) or as one-pot synthesis (Method 2). As mentioned, lithium intercalation can be applied to exfoliate bulk $\mathrm{MoS}_{2}$ into $1 \mathrm{~T}-\mathrm{MoS}_{2}$. However, a prolonged heating above the $95^{\circ} \mathrm{C}$ threshold using hydrothermal techniques on non-stabilised $1 \mathrm{~T}$ $\mathrm{MoS}_{2}$ would reconvert the structure from metallic 1T- to semiconducting $2 \mathrm{H}-\mathrm{MoS}_{2}$. For the one-pot hydrothermal/solvothermal synthesis, nanosheets are being built-up unit by unit through reactions between the Mo and $S$ 
precursors. The heteroatoms are then incorporated onto the asprepared nanosheets afterwards. Since the six-coordinated Mo atoms are well located inside the intact $\mathrm{MoS}_{2}$ unit cells, the incoming heteroatoms dopants during the incorporation process are less likely to penetrate into the outer S layer and substitute the Mo atom. Therefore, they are placed at the Mo atop sites or $\mathrm{S}$ substitution sites unless extra treatment such as high temperature annealing is applied.

For the second route, the one pot bottom-up synthetic approach can also be adopted to directly synthesise SAC-MoS through the reactions between Mo, S, and the dopant precursors. The heteroatoms can be embedded inside the $\mathrm{MoS}_{2}$ nanosheets as a result due to its presence during the unit by unit construction of $\mathrm{MoS}_{2}$ nanosheets. However, it should be noted that a precise control on the molar concentration for the three precursors is vital for obtaining monolayer nanosheets confined with single heteroatoms within the tolerance of the thermodynamic maximum limits. An excess amount of single metal atoms dopants may result in the formation of clusters or nanoparticles ${ }^{28}$ while an insufficient amount of Mo or $\mathrm{S}$ sources can lead to the creation of surface defects ${ }^{30}$.

Several selected publications listed in table 1 (Entry 1, 3, 5) adopted the first route to synthesise the SAC-MoS 2 . (Figure 1, Method 1) Lithium intercalation was applied to obtain 1T-MoS nanosheets. The delicate $1 T$ polytype was then preserved when the as-prepared nanosheets undergo doping under sonochemical reaction at ambient condition to prevent the conversion back to $2 \mathrm{H} .{ }^{29}$ The same hydrothermal/ solvothermal techniques are also being applied in one pot synthesis of SACMoS $_{2}$. (Table 1, Entry 2, 3, 4, 6, 7) (Figure 1, Method 3) While the reaction temperature in all cases are at least above $180^{\circ} \mathrm{C}$, the metal dopants embedded into the as-grown $\mathrm{MoS}_{2}$ can provide extra kinetic stability to prevent the conversion of $1 \mathrm{~T}$ polytypes. However, this only applies to a certain extent as the $\mathrm{MoS}_{2}$ nanosheets grown at a high temperature of $400^{\circ} \mathrm{C}$, which will still drive to $2 \mathrm{H}$ polytype with a multiple-layers thickness. Therefore, the synthetic condition also plays a crucial role in determining the resulting polytypes. Overall, the doping concentration, doping position, and the polytypes largely depends on the synthetic route and condition. Since there are pros and cons for each discussed synthetic method, the choice should depend on the structure of the SAC-MoS 2 material desired as it ultimately determines the catalytic performance.

\section{Catalytic Performance of SAC-MoS 2}

The electrocatalytic performance of catalysts can be evaluated by several key parameters. An ideal HER catalyst should possess low overpotential, low Tafel slope, and high stability while the synthetic cost should remain minimal. In theory, HER can take place at a cell voltage of oV but actually requires extra potential to initiate the reaction due to the resistance in the real electrochemical system. This extra potential is called overpotential ( $\eta$ ), which has been commonly used for assessing the strength of the catalyst to reduce the activation energy of HER. However, it should be careful that the value can also be largely affected by the $\mathrm{pH}$ value of the electrolyte and the molar concentration of the analytes. As a result, the research highlighted in this article mainly employed an acidic medium in their electrochemical systems. Tafel slope is another characteristic indicator to study the activity of the electrocatalyst. It reflects how the logarithm of the current

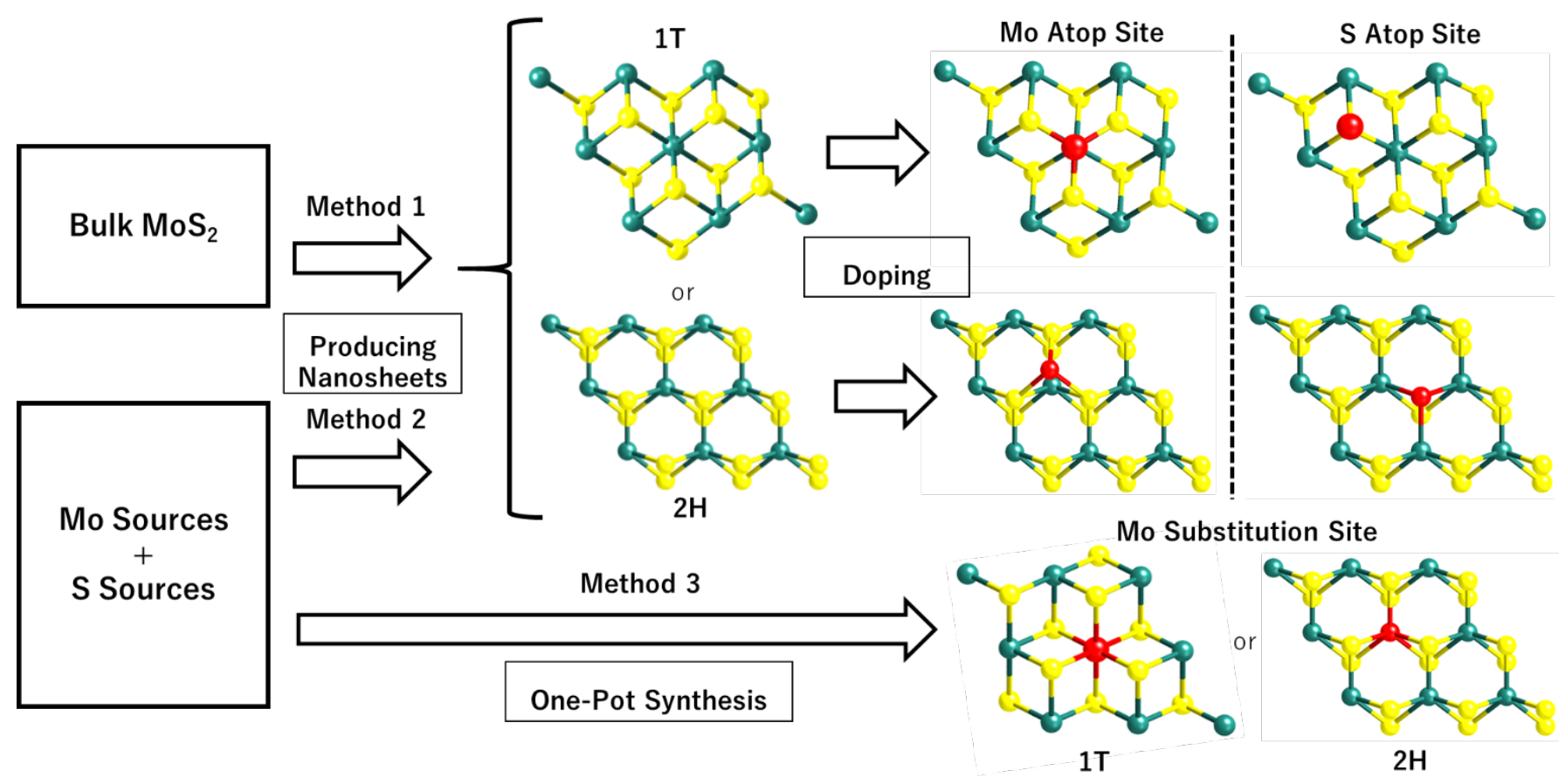

Figure 1. Summary of the synthetic routes of SAC-MoS 2 adopted in recent highlighted publications. Method 1 uses the top-down exfoliation of bulk MoS $\mathrm{S}_{2}$ to create MoS $\mathrm{S}_{2}$ nanosheets while method 2 uses the bottom-up hydrothermal/solvothermal reactions to make the nanosheets directly from Mo and $\mathrm{S}$ sources. The pure MoS $\mathrm{created}$ with method 1 and method 2 will then be doped by the heteroatoms to form SAC-MoS ${ }_{2}$ Method 3 uses one-pot synthesis of the SAC-MoS 2 via hydrothermal/solvothermal reactions. For all the MoS structural models shown in the above figure, the green, yellow, and red spheres represent Mo atoms, $\mathrm{S}$ atoms, and dopant atoms respectively. 
density varies with the overpotential: the derived Tafel slope is

\begin{tabular}{|c|c|c|c|c|}
\hline Entry & Catalyst & $\begin{array}{c}\text { Overpotential ( } \eta) \\
\left(\mathrm{mV} @ 10 \mathrm{~mA} / \mathrm{cm}^{2}\right) \text { Vs RHE }\end{array}$ & $\begin{array}{l}\text { Tafel Slope } \\
\text { (mV/ decade) }\end{array}$ & Ref. \\
\hline $1 \mathrm{~A}$ & $\mathrm{MoS}_{2}$ & $\sim-375 \mathrm{mV}$ & 186 & 25 \\
\hline $1 \mathrm{~B}$ & $\mathrm{MoS}_{2}$ & $\sim-250 \mathrm{mV}$ & 40 & 25 \\
\hline $2 \mathrm{~A}$ & $\mathrm{Co}-\mathrm{MoS}_{2}$ & $\sim-285 \mathrm{mV}$ & $N / A$ & 26 \\
\hline $2 \mathrm{~B}$ & Pt-MoS 2 & $\sim-185 \mathrm{mV}$ & 96 & 26 \\
\hline 3 & $\begin{array}{c}\mathrm{Co}^{\mathrm{CMoS}} \mathrm{M}_{2} \\
\text { with S defects }\end{array}$ & $-300 m V$ & 92 & 27 \\
\hline 4 & $\mathrm{Pd}-\mathrm{MoS}_{2}$ & $-89 m V$ & 62 & 28 \\
\hline $5 A$ & $\mathrm{Pt}-\mathrm{MoS}_{2}$ & $-223 m V$ & 57 & 29 \\
\hline $5 B$ & $\mathrm{Pd}-\mathrm{MoS}_{2}$ & $-140 \mathrm{mV}$ & 50 & 29 \\
\hline 6 & $\mathrm{P}-\mathrm{MoS}_{2}$ & $-43 m V$ & 34 & 30 \\
\hline 7 & $\mathrm{~N}-\mathrm{MoS}_{2}$ & $-168 m V$ & 41 & 31 \\
\hline
\end{tabular}

inversely proportional to the charge transfer coefficient. Thus, the small value of Tafel slope indicates a high charge transfer ability. ${ }^{51}$ Tafel slope can also be used to reveal the rate determining step (RDS) of the reaction. It is well known that reaction mechanism of hydrogen evolution will be changed under different $\mathrm{pH}$ condition. Acid solutions are generally used as electrolytes as they show higher ionic conductivity and no carbonate will be formed in the process, as compared with alkaline medium. ${ }^{52}$ There are the three fundamental reaction steps involved in the overall HER (Figure 2) in acidic medium. The Volmer step describes the generation of an adsorbed hydrogen atom $\left(H_{a d s}^{*}\right)$ at the catalyst $\left(\mathrm{H}^{+}(\mathrm{aq})+\mathrm{e}^{-} \rightarrow H_{a d s}^{*}\right)$.

The reaction then proceeds with either via the Tafel step or the Heyrovsky step, which is usually dependent on the amount of $H_{a d s}^{*}$ available. In the Tafel step, two absorbed hydrogen atoms recombine to yield $\mathrm{H}_{2}$ gas $\left(2 \mathrm{H}_{a d s}^{*} \rightarrow \mathrm{H}_{2(\mathrm{~g})}\right)$. Whereas for the Heyrovsky step, $H_{a d s}^{*}$ will interact with another electron and proton pair to form $\mathrm{H}_{2}\left(H_{a d s}^{*}+\mathrm{H}^{+}{ }_{(\mathrm{aq})}+\mathrm{e}^{-} \rightarrow \mathrm{H}_{2}(\mathrm{~g})\right)$. A Tafel slope value of 120,40 , and $30 \mathrm{mv} /$ decade indicates that the RDS are characterised by the Volmer step, Heyrovsky step, and the Tafel step, respectively for $\mathrm{MoS}_{2}$ based materials. ${ }^{53}$ Thus, through obtaining the Tafel slope values, it can be predicted that which reaction pathway, Volmer-Heyrovsky reaction or Volmer-Tafel reaction are playing the important role in affecting the RDS.
Table 1 lists out the overpotentials and the Tafel slopes values of different SAC-MoS ${ }_{2}$ from the selected publications. It can be observed that undoped $\mathrm{MoS}_{2}$ with $1 \mathrm{~T}$ polytypes have a much smaller Tafel slope value (40mv/decade) than that of semiconducting $2 \mathrm{H}$ counterparts $(180 \mathrm{mv} / \mathrm{dec})$ (Table 2, Entry $1 \mathrm{~A}$ and $1 \mathrm{~B})$. As explained earlier, SAC-MoS 2 nanosheets with $1 \mathrm{~T}$ polytypes is metallic and surface-active. $H_{a d s}^{*}$ will be readily formed at the basal plane and the Volmer step will not be the $\mathrm{RDS}$ in this case. In contrast, it is difficult for $\mathrm{H}$ adsorption to be taken place on basal plane of $2 \mathrm{H} / 1 \mathrm{H}-\mathrm{MoS}_{2}$. The overall reaction rate is hindered by insufficient $\mathrm{H}$ adsorption, which also suggested why the overpotential of $2 \mathrm{H}-\mathrm{MoS}_{2}$ is larger than that of $1 \mathrm{~T}$. As a result, the metallic $1 \mathrm{~T}-\mathrm{MoS}_{2}$ based structures appear to be more useful than 2-H counterparts for the electrochemical hydrogen production due to lower impedance encountered, giving higher energy conversion. However, the long-term stability of this structure under practical conditions may have to be ascertained for applications in electrolyser.

To understand the effect of single metal dopants on $\mathrm{MoS}_{2}$ for HER, Deng et al. were among the pioneer groups to conduct electrochemical scan tests of $2 \mathrm{H}-\mathrm{MoS}_{2}$ doped with several types of single transition metal atoms ( $\mathrm{Pt}, \mathrm{Co}$, and $\mathrm{Ni}$ ) at $\mathrm{Mo}$ substitution sites for HER (Table 2, Entry 2). ${ }^{26}$ According to the experimental results, the $\mathrm{Pt}$ doped one exhibited the best catalytic performance among three with the smallest overpotential of $-185 \mathrm{mV}$ at $10 \mathrm{~mA} / \mathrm{cm}^{2}$. The Density of State (DOS) simulation provided an explanation that the introduction of $\mathrm{Pt}$ atoms into the Mo substitution site induced more hybridized electronic states to occur near the Fermi level, which enhanced $\mathrm{H}$ adsorption ability of basal $\mathrm{S}$ atoms and thus the catalytic performance for HER. (Figure $2 a$ and $b$ )

Density functional theory (DFT) calculations are also applied to calculate the Gibbs energy for hydrogen adsorption $\left(\Delta \mathrm{G}_{\mathrm{H}^{*}}\right)$ of the catalytic or electrocatalytic system when doped with different single transition metal atoms in order to predict the overall trend. According to the Sabatier principle, the $\mathrm{H}$ adsorption process is optimal when $\Delta \mathrm{G}_{\mathrm{H}^{*}}$ is close to zero. ${ }^{54}$

(a)

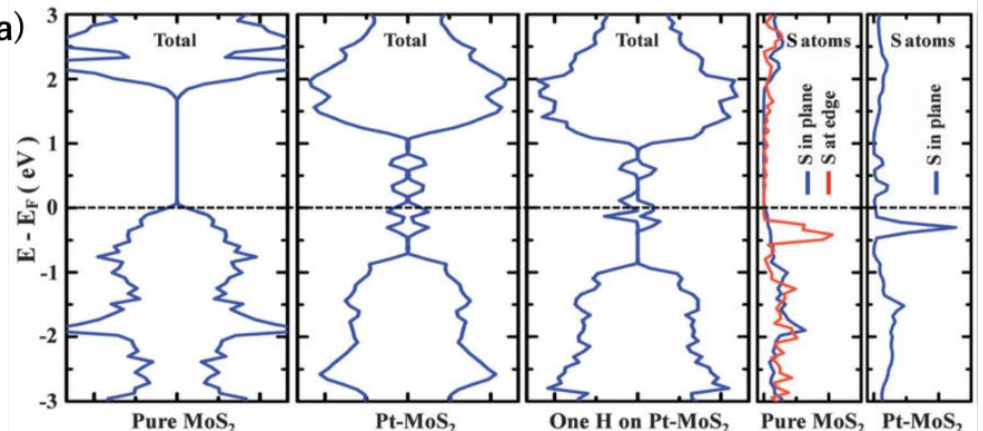

(b)
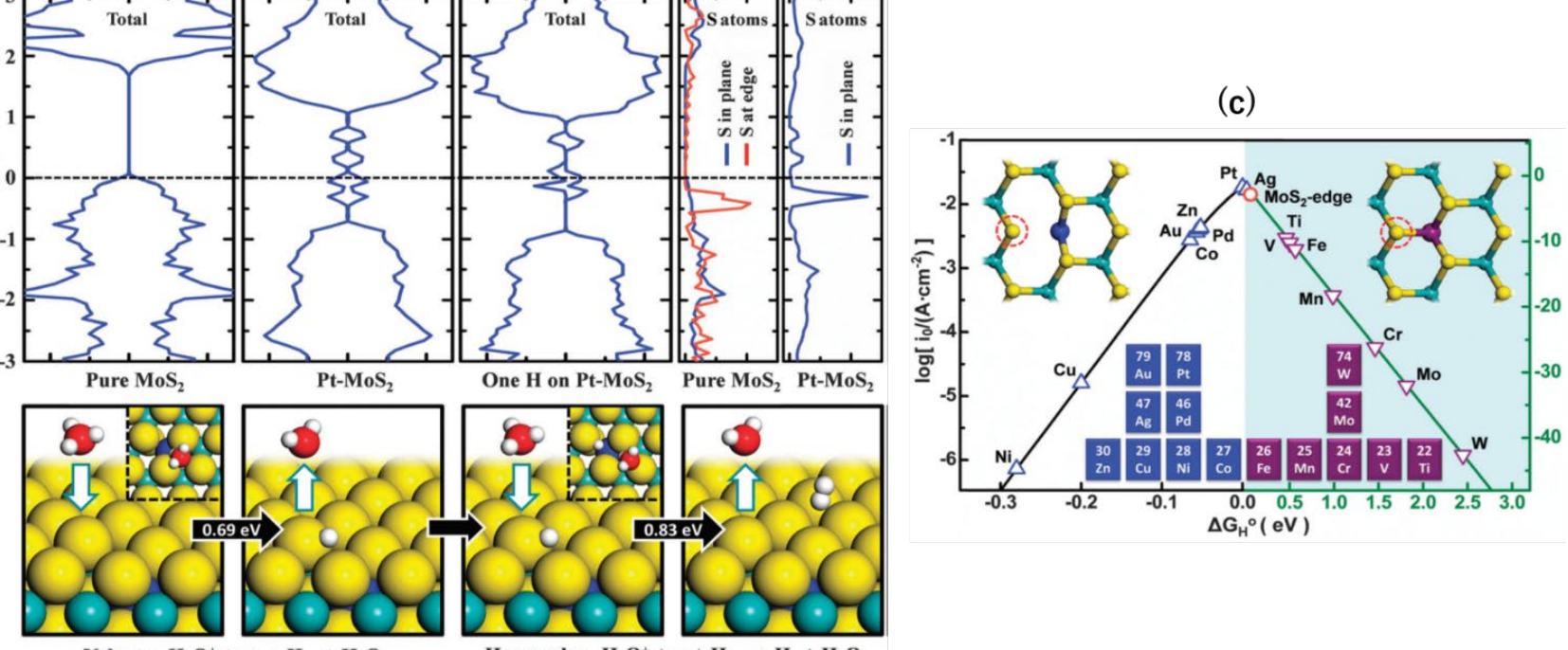

Volmer: $\mathrm{H}_{3} \mathrm{O}^{+}+\mathrm{e}^{-} \rightarrow \mathrm{H}_{\text {ad }}+\mathrm{H}_{2} \mathrm{O}$

Heyrovsky: $\mathrm{H}_{3} \mathrm{O}^{+}+\mathrm{e}^{-}+\mathrm{H}_{\text {ad }} \rightarrow \mathrm{H}_{2}+\mathrm{H}_{2} \mathrm{O}$

Figure 2 (a) DOS of $\mathrm{MoS}_{2}, \mathrm{Pt}-\mathrm{MoS}_{2}, \mathrm{H}$ adsorbed Pt-MoS . The dashed line represents the fermi level (b) HER process through Volmer-Heyrovsky pathway on a Pt-MoS catalyst. (c) Volcano plot of $2 \mathrm{H}-\mathrm{MoS}_{2}$ doped with different transition metal at the Mo substitution site. ${ }^{26}$ 

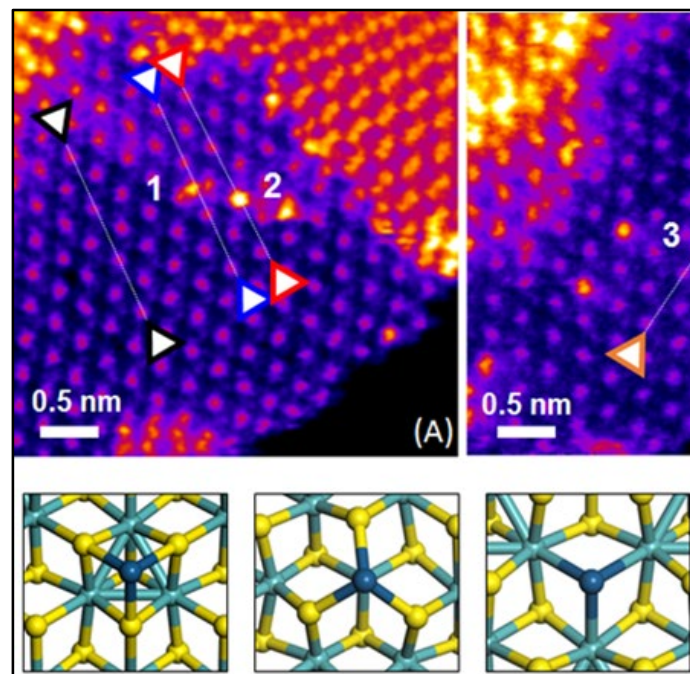

1. Pt on $S^{\prime}$
atoms
2. Pt on $\mathrm{Mo}$ atoms

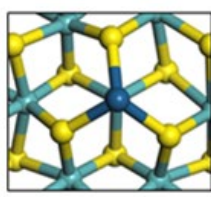

3. Pt in S

Vacancy
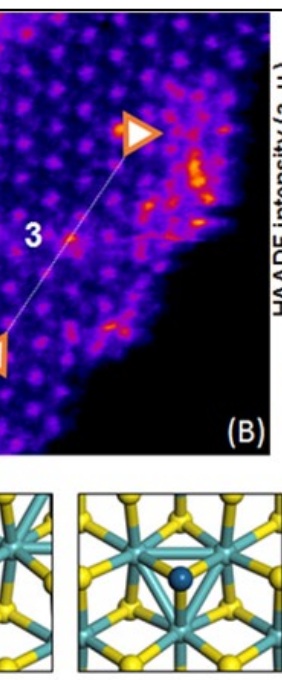

4. Pt on S atoms

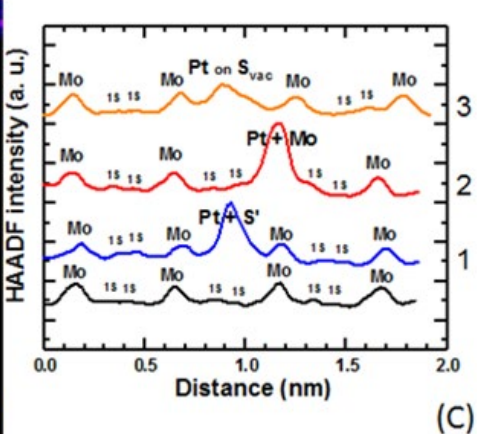

(C)

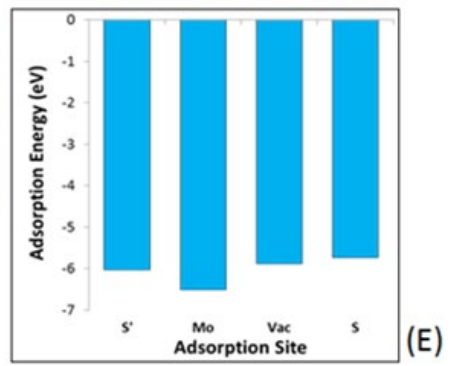

Figure 3. Structural analysis of $1 \% \mathrm{Pd}-\mathrm{MoS}_{2}$ and $\mathrm{MoS}_{2}$. (a) Pd-3d XPS and (b) XRD spectrum of the 1\%Pd-MoS 2 . Fourier transform of the k3-weighted (c) Pd K-edge and (d) Mo Kedge of the EXAFS spectra. (e) Dark-field scanning transmission electron microscopy image of the $1 \% \mathrm{Pd}-\mathrm{MoS}_{2}$. Blue and yellow dots represent Mo and $\mathrm{S}$ atoms respectively. The white line is the scale bar of $1 \mathrm{~nm}$. (f) ESR spectra and (g) Raman spectra of $1 \% \mathrm{Pd}-\mathrm{MoS}_{2}$ and $\mathrm{MoS}_{2}$. (h) Bode spectra with a frequency range from $0.1 \mathrm{~Hz}$ to $10 \mathrm{kHz}$ and an amplitude of $5.0 \mathrm{mV} .{ }^{28}$

When the value of $\Delta \mathrm{G}_{\mathrm{H}^{*}}$ is too negative, the hydrogen atom will bind too strongly onto the surface, which inhibits the subsequent desorption process for the formation of $\mathrm{H}_{2}$ gas until thermal or electrical energy is supplied. On the contrary, if the value of $\Delta \mathrm{G}_{\mathrm{H}^{*}}$ is too positive, the catalyst surface will not favour the adsorption process for either the catalytic or electrocatalytic processes. It should be noted that this principle offers good predictive values to metallic phases with no impedance or diffusional limitations imposed between the active sites. Pt is known to be at the apex of the Sabatier volcano for $\mathrm{H}$ species. On the other hand, for composite materials such as SAC-MoS 2 one should consider the composite surface for the $\mathrm{H}$ interactions. For unmodified single molecular $2 \mathrm{H}-\mathrm{MoS}_{2}$ monolayers, the value of Tafel slope ( $96 \mathrm{mv} /$ decade) indicated that $\mathrm{H}$ adsorption is predominately the RDS for overall HER. A plot of the reaction rate of $2 \mathrm{H}-\mathrm{MoS}_{2}$ when doped with different metal atoms (in terms of exchange current density) against their $\Delta \mathrm{G}_{\mathrm{H}^{*}}$ in HER was shown to have a volcano shape with Pt at the apex, indicating the dominant role of $\mathrm{Pt}$ than the $2 \mathrm{H}-\mathrm{MoS}_{2} .{ }^{26}$ (Figure 2c) The excellent experimental results of Co-doped $\mathrm{MoS}_{2}$ compared with other metals further supported the volcano plot. This suggests that this inexpensive non-noble metal can be effectively used without much activity compromise than using Pt. However, this work did not give a satisfactory explanation for the surprisingly poor $\mathrm{Ni}$ performance, which is electronically similar to Co atom as they are neighbour elements to each other. Another independent work also studied how the single transition metal atoms ( $\mathrm{Co}, \mathrm{Ni}$, $\mathrm{Ag}, \mathrm{Cu}$ ) doped at the Mo atop sites would affect the semiconducting $2 \mathrm{H}-\mathrm{MoS}_{2}$, but with a somewhat different conclusion drawn. The Co-MoS 2 was confirmed to be the best HER catalyst among four transition metals with a Tafel slope value of $92 \mathrm{mv} /$ decade and an overpotential of $-300 \mathrm{mV}$ at $10 \mathrm{~mA} / \mathrm{cm}^{2}$ (Table 2, Entry 3). ${ }^{27}$ The DOS calculations suggested that Co dopants help shifting the conduction band close to the fermi level, which ultimately makes the surrounding $\mathrm{S}$ atoms at the basal planes better active sites for $\mathrm{H}$ adsorption. Also, no nice volcano relationship was noted when considering theoretical electronic factors of the metal dopers. Extended Xray absorption fine structure (EXAFS) was in fact conducted to study the chemical environment of the dopants. Interestingly, it was found that Co atoms anchored at the Mo atop site did not form a Co-Mo bond due to geometric constraint whereas the $\mathrm{Ni}$-Mo formed negatively influence the local $\mathrm{S}$ atoms in terms of the $\mathrm{H}$ adsorption ability. Thus, this clearly demonstrates that the metal doper can have different interaction with the single molecular layer $\mathrm{MoS}_{2}$. Therefore, basing solely on the electronic factor it is difficult to predict the trend without considering the precise position of the dopers and the steric constraints of the atoms in the composite structures. Regardless, the two pieces of research have interestingly shown that even when the same metal atoms are doped, the different doping position (Mo substitution site and Mo atop site) will largely affect the electronic properties of the resulting SAC- $\mathrm{MoS}_{2}$ with $1 \mathrm{H} / 2 \mathrm{H}$ polytypes.

For SAC-MoS 2 with $1 \mathrm{~T}$ polytypes, luo et al. suggested a significantly much stronger interaction between transition metal doper and the $1 \mathrm{~T}-\mathrm{MoS}_{2}$ support. They showed that the inclusion of $1 \% \mathrm{Pd}$ atoms into the $1 \mathrm{~T}$ structure can dramatically boost the HER activity (Table 2, Entry 4 ). ${ }^{28}$ It is confirmed by Xray photoelectron spectroscopy (XPS), $X$-ray powder diffraction and EXAFS that Pd atoms were successfully grown at the Mo substitution sites (Figure 3). It is also possible to help create $S$ defects during the synthesis. DFT calculations suggested that 

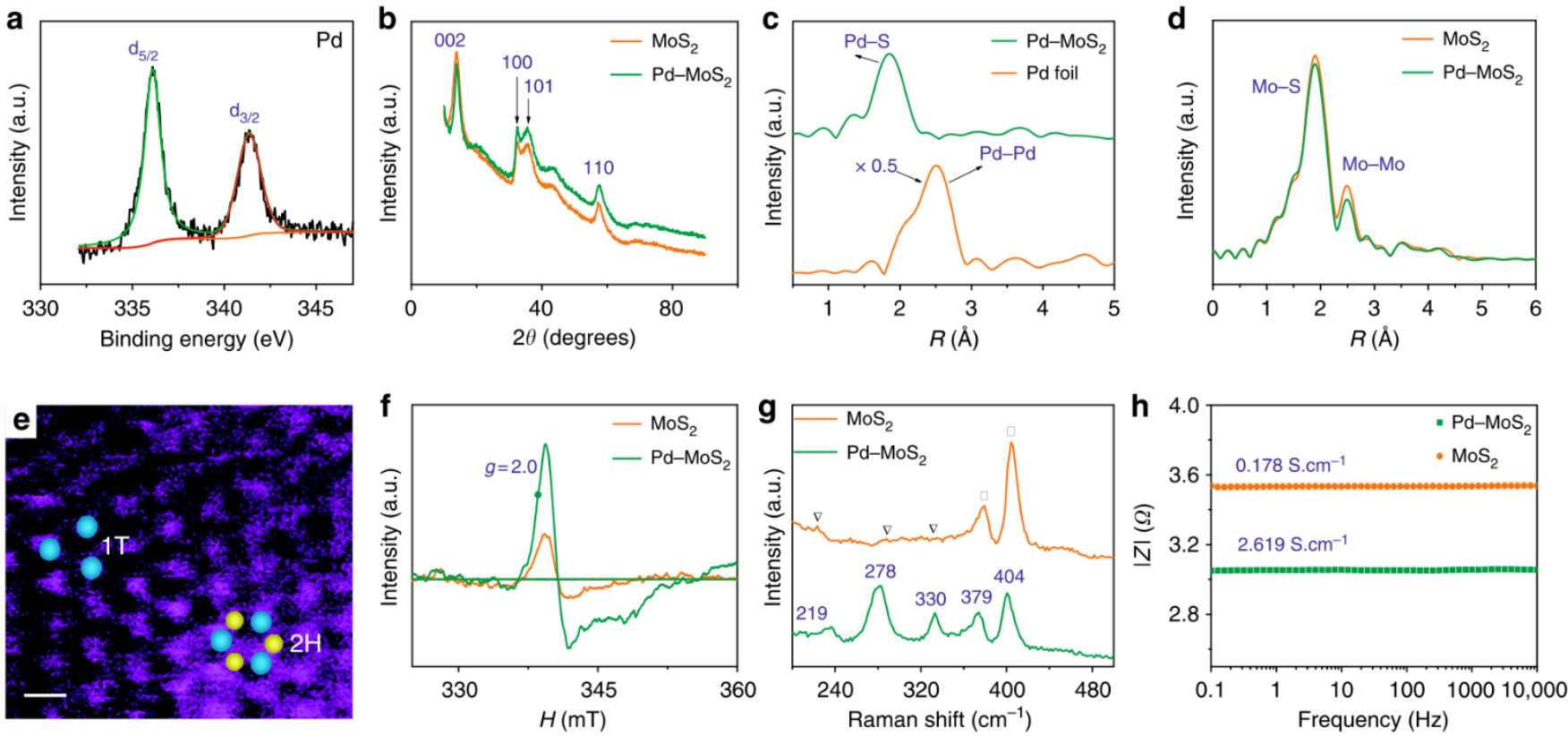

Figure 4. (a) and (b) HAADF-STEM of Pt-1T-SMoS 2 . (c) ADF intensity line profiles taken along the corresponding coloured line of Black, Blue, Red, and Orange at <110> direction. (d) The purposed model of the Pt atom doping at 4 different adsorption sites. Numbers 1,2 and 3 correspond to the experimental observed Pd position shown in (a) and (b). (e) Adsorption Energy for Pt atom on each purposed adsorption site. ${ }^{29}$

the $\Delta \mathrm{G}_{\mathrm{H}}$ of the $\mathrm{S}$ adjacent to $\mathrm{Pd}\left(\mathrm{Pd}-\mathrm{S}^{*}-\mathrm{Mo}\right)$ exhibited an almost thermoneutral value of $-0.02 \mathrm{eV}$. Similar to aforementioned metal atoms doped on $2 \mathrm{H}-\mathrm{MoS}_{2}$, the major function of $\mathrm{Pd}$ atoms help activating the adjacent $\mathrm{S}$ atoms ( $\mathrm{Pd}-\mathrm{S}^{*}-\mathrm{Mo}$ ) at the basal plane of $1 \mathrm{~T}-\mathrm{MoS}_{2}$. As suggested by the durability test, the highly stable $\mathrm{Pd}-\mathrm{S}$ bonds also provide overall stability, which is another important criterion for a good heterocatalyst. However, it also should be noted that since the metastable 1T polytype is just a distorted form of $2 \mathrm{H}$ polytypes, both $1 \mathrm{~T}$ and $2 \mathrm{H}$ polytypes will always coexist upon the incorporation of heteroatoms. Most key research in the literature have tended to neglect this important factor as the degree of coexistence (1T:2H ratios) were usually not provided nor addressing their correlation to activity. Another independent research works also studied the catalytic performance of Pd doped on 1T-MoS 2 . The High-angle annular dark-field scanning transmission
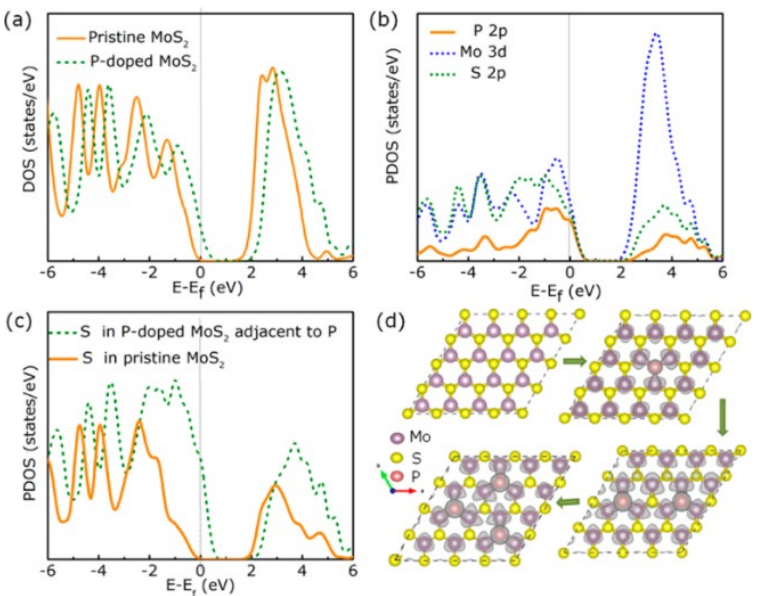

Figure 5. (a) Total DOS of pure $\mathrm{MoS}_{2}$ and P doped $\mathrm{MoS}_{2}$. (b) Partial DOS of P-MoS 2. (c) Partial DOS for $S$ atoms. The vertical grey lines represent the fermi level. (d) Partial charge density of $\mathrm{P}-\mathrm{MoS}_{2}$ when doped with 0-3 $\mathrm{P}$ atoms. electron microscopy (HAADF-STEM, Figure 4) combined with other structural characterisation techniques and computational calculations suggested that the $\mathrm{Pd}$ atoms are doped at the Mo atop site rather than the Mo substitution site. ${ }^{29}$ The Tafel slope analysis and DFT modelling reconfirmed that the $\mathrm{H}$ adsorption was readily facilitated by the $1 \mathrm{~T}$ surface and thus Volmer step is not the RDS. More importantly, the incorporated Pd atoms here could act as the active sites to accelerate the rate limiting recombination step (Heyrovsky step or Tafel step) to form hydrogen gas. Overall, it was suggested that $\mathrm{H}$ adsorption would preferably be taken place on the $1 \mathrm{~T}$ surface. The $H_{a d s}^{*}$ then hopped to the single Pd atoms to be recombined to $\mathrm{H}_{2}$. The difference in the roles of the Pd atoms for HER from these two different studies probably originates from the two different doing positions due to their difference in synthetic methods used. The Pd atoms substituting Mo atoms usually formed stable Pd-S bonds which may offer steric hindrance to prevent any interactions with the $H_{a d s}^{*}$ while uncoordinated Pd atoms at the Mo atop site are electronically and spatially freely to undergo redox reactions, making $\mathrm{Pd}-1 \mathrm{~T}-\mathrm{MoS}_{2}$ a bifunctional catalyst for HER.

It is interesting to note that the non-metal $\mathrm{P}$ atoms doped $1 \mathrm{~T} \mathrm{MoS}$ nanosheets prepared by Liu and co-workers exhibited a small Tafel slope of $34 \mathrm{mV} /$ decade with an excellent overpotential of $43 \mathrm{mV}$ at $10 \mathrm{~mA} / \mathrm{cm}^{2}$ (Table 2, Entry 6). Its catalytic performance is comparable to that of $\mathrm{Pt}$ doped graphene (with an overpotential of $45 \mathrm{mV}$ and a Tafel slope of $29 \mathrm{mv} /$ decade) which is considered to be one of the best SAC electrocatalysts reported. ${ }^{55}$ The DFT calculation suggested the possibility that the $\mathrm{P}$ atoms incorporated at $\mathrm{S}$ substitution sites ( $\mathrm{S}$ vacancy sites) could both act as new main active sites for $\mathrm{H}$ adsorption and activate the basal $\mathrm{S}$ atoms of $\mathrm{MoS}_{2}$ at the same time. This offers new opportunity to develop non-metal based 
catalyst with no metal leaching issues during electrolysis of water at high potentials. The DOS and partial DOS simulations also suggested that more electronic states are shifted near the Fermi level after introducing $P$ atoms (Figure 5), which ultimately increased the electrical conductivity at the basal plane for enhanced HER. In contrary, non-metal $\mathrm{N}$ atoms doped at Mo substitution site once again did not directly participate in HER but helped activate the surrounding $\mathrm{S}$ atoms to be more active for the $\mathrm{H}$ adsorption. (Table 2, Entry 7) Similarly, more DOS can be observed at Fermi level when $\mathrm{N}$ atoms were embedded into $\mathrm{MoS}_{2}$, which also increases the electrical conductivity of basal sites. This once again reinforces the emphasis on how important the nature of the doper and the doping position can be when tailoring the SAC-MoS $S_{2}$ for hydrogen reduction.

\section{Conclusions}

Effective use of catalyst component to convert substrate molecule to product is important in catalysis. As stated, the use of SACs on 2D materials is a hot topic of research due to their high characteristic surface area to volume ratios and the electronic and steric influence on catalytic active atoms can be made clearer over these defined nanostructures. However, at this early stage, there are still limited amounts of high-quality data with corresponding details in experimental analysis, and computational calculations to lead to atomic understanding of catalyst structure and activity relationship.

Here, through reviewing some selected key publications of SAC-MoS 2 for HER, we can draw some conclusions with new insights on the otherwise commonly used term 'metal-support interactions' in heterogeneous catalysis. Also, understanding HER reaction over catalyst surface for $\mathrm{H}_{2}$ production from water is also very important in developing new catalytic technologies to harness renewable energies. It is shown that the polytypes of 2D $\mathrm{MoS}_{2}$ (the specific support material with characteristic structure and properties), the choice of the single heteroatoms (i.e. both metal or non-metal atoms), and the doping position (define site for specific electronic and steric perturbation) are the three most important factors for deciding the catalytic performance of the resulting SAC-MoS 2 in HER. Conventional electrochemical scan tests and volcano plot can be used to gauge which metal dopants on $2 \mathrm{H}-\mathrm{MoS}_{2}$ support could potentially help enhancing the overall HER performance. DFT modelling is important to characterise the electronic and steric properties of the atoms as catalyst as well as the influence from the support. However, such trend can subtly be changed if the chemical nature of dopers, relative sizes, and dwelling positions are altered. As a result, atomic positions on defined structures with defined interactions are required to gain further understanding of structure-activity relationships. Specifically to $\mathrm{HER}$, the heteroatoms that replace Mo atoms can activate the adjacent in-plane $\mathrm{S}$ atoms for $\mathrm{H}$ adsorption which ultimate enhance the ability for $\mathrm{H}$ adsorption. They are anticipated to be more stable and durable in position since they are stably embedded into the $\mathrm{MoS}_{2}$ structure. Whereas heteroatoms doping at the Mo atop sites or $\mathrm{S}$ vacancy sites though interacting with the outer S layers can act as the active sites themselves and also activate the surrounding $S$ through electronic perturbation for HER. In the case where the basal $\mathrm{S}$ atoms have already been activated for $\mathrm{H}$ adsorption by other reasons, for example the heteroatoms are doped on a S defect rich $1 \mathrm{~T}-\mathrm{MoS}_{2}$, the heteroatoms may become the active sites for recombination of $H_{a d s}^{*}$ to $\mathrm{H}_{2}$ if preferable. Overall, all these parameters could be tailored by choosing the correct synthetic methods, which

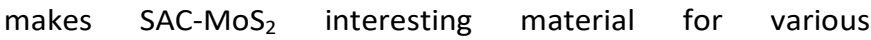
electrochemical applications. This also gives insight into developing other 2D nanomaterials confined with different heteroatoms for various catalytic uses.

\section{Conflicts of interest}

There are no conflicts to declare.

\section{Notes and references}

1 W. Choi, I. Lahiri, R. Seelaboyina and Y. S. Kang, Crit. Rev. Solid State Mater. Sci., 2010, 35, 52-71.

2 D. Akinwande, C. J. Brennan, J. S. Bunch, P. Egberts, J. R. Felts, H. Gao, R. Huang, J.-S. Kim, T. Li, Y. Li and others, Extrem. Mech. Lett., 2017, 13, 42-77.

3 M. Chhowalla, H. S. Shin, G. Eda, L. J. Li, K. P. Loh and H. Zhang, Nat. Chem., 2013, 5, 263-275.

H. Pan, Sci. Rep., 2014, 4, 1-6.

5 M. Chhowalla, Z. Liu and H. Zhang, Chem. Soc. Rev., 2015, 44, 2584-2586.

6

A. Yusuf, C. Snape, J. He, H. Xu, C. Liu, M. Zhao, G. Z. Chen, B. Tang, C. Wang, J. Wang and S. N. Behera, Catal. Rev. Sci. Eng., 2017, 59, 189-233.

7 S. Royer and D. Duprez, ChemCatChem, 2011, 3, 24-65.

8 M. Khazaei, M. Arai, T. Sasaki, C. Y. Chung, N. S. Venkataramanan, M. Estili, Y. Sakka and Y. Kawazoe, Adv. Funct. Mater., 2013, 23, 2185-2192.

9 J. Xie and Y. Xie, Chem. - A Eur. J., 2016, 22, 3588-3598.

10 D. Kong, H. Wang, J. J. Cha, M. Pasta, K. J. Koski, J. Yao and Y. Cui, Nano Lett., 2013, 13, 1341-1347.

11 Y. Zhang, Q. Ji, G. F. Han, J. Ju, J. Shi, D. Ma, J. Sun, Y. Zhang, M. Li, X. Y. Lang, Y. Zhang and Z. Liu, ACS Nano, 2014, 8, 8617-8624.

12 H. Nan, Z. Wu, J. Jiang, A. Zafar, Y. You and Z. Ni, J. Phys. D. Appl. Phys., 2017, 50.

13 M. R. Islam, N. Kang, U. Bhanu, H. P. Paudel, M. Erementchouk, L. Tetard, M. N. Leuenberger and S. I. Khondaker, Nanoscale, 2014, 6, 10033-10039.

14 L. Tao, X. Duan, C. Wang, X. Duan and S. Wang, Chem. Commun., 2015, 51, 7470-7473.

15 H. Li, C. Tsai, A. L. Koh, L. Cai, A. W. Contryman, A. H. Fragapane, J. Zhao, H. S. Han, H. C. Manoharan, F. AbildPedersen, J. K. Nørskov and X. Zheng, Nat. Mater., 2015, 15, 48-53.

16 H. Li, A. W. Contryman, X. Qian, S. M. Ardakani, Y. Gong, X. Wang, J. M. Weisse, C. H. Lee, J. Zhao, P. M. Ajayan, J. Li, H. C. Manoharan and X. Zheng, Nat. Commun., 2015, 6. 
17 Y. Tan, P. Liu, L. Chen, W. Cong, Y. Ito, J. Han, X. Guo, Z. Tang, T. Fujita, A. Hirata and M. W. Chen, Adv. Mater., 2014, 26, 8023-8028.

18 B. Qiao, A. Wang, X. Yang, L. F. Allard, Z. Jiang, Y. Cui, J. Liu, J. Li and T. Zhang, Nat. Chem., 2011, 3, 634-641. G. Vilé, D. Albani, M. Nachtegaal, Z. Chen, D. Dontsova, M. Antonietti, N. López and J. Pérez-Ramírez, Angew. Chemie Int. Ed., 2015, 54, 11265-11269. X.-F. Yang, A. Wang, B. Qiao, J. Li, J. Liu and T. Zhang, Acc. Chem. Res., 2013, 46, 1740-1748. A. Alarawi, V. Ramalingam and J. H. He, Mater. Today Energy, 2019, 11, 1-23. C. Zhu, S. Fu, Q. Shi, D. Du and Y. Lin, Angew. Chemie - Int. Ed., 2017, 56, 13944-13960. K. C. Kwon, J. M. Suh, R. S. Varma, M. Shokouhimehr and H. W. Jang, Small Methods, 2019, 3, 1-20. Q. H. Wang, K. Kalantar-Zadeh, A. Kis, J. N. Coleman and M. S. Strano, Nat. Nanotechnol., 2012, 7, 699-712. D. Voiry, M. Salehi, R. Silva, T. Fujita, M. Chen, T. Asefa, V. B. Shenoy, G. Eda and M. Chhowalla, Nano Lett., 2013, 13, 6222-6227. J. Deng, H. Li, J. Xiao, Y. Tu, D. Deng, H. Yang, H. Tian, J. Li, P. Ren and X. Bao, Energy Environ. Sci., 2015, 8, 15941601. T. H. M. Lau, X. Lu, J. Kulhavý, S. Wu, L. Lu, T. S. Wu, R. Kato, J. S. Foord, Y. L. Soo, K. Suenaga and S. C. E. Tsang, Chem. Sci., 2018, 9, 4769-4776. Z. Luo, Y. Ouyang, H. Zhang, M. Xiao, J. Ge, Z. Jiang, J. Wang, D. Tang, X. Cao, C. Liu and W. Xing, Nat. Commun., 2018, 9, 1-8.

T. H. M. Lau, S. Wu, R. Kato, T. S. Wu, J. Kulhavý, J. Mo, J. Zheng, J. S. Foord, Y. L. Soo, K. Suenaga, M. T. Darby and S. C. E. Tsang, ACS Catal., 2019, 9, 7527-7534. P. Liu, J. Zhu, J. Zhang, P. Xi, K. Tao, D. Gao and D. Xue, ACS Energy Lett., 2017, 2, 745-752. R. Li, L. Yang, T. Xiong, Y. Wu, L. Cao, D. Yuan and W. Zhou, J. Power Sources, 2017, 356, 133-139. M. Acerce, D. Voiry and M. Chhowalla, Nat. Nanotechnol., 2015, 10, 313-318.

3 F. Wypych and R. Schollhorn, J. CHEM. SOC.. C'HEM. COMMUN., 1992, 1386-1388. D. B. Putungan, S. Lin and J. Kuo, 2015, 2, 21702-21708. J. C. Phys, 2016, 174702. T. F. Jaramillo, K. P. Jørgensen, J. Bonde, J. H. Nielsen, S. Horch and I. Chorkendorff, Science, 2007, 317, 100-102. H. Tang and S. Roy Morrison, Thin Solid Films, 1993, 227, 90-94. M. A. Lukowski, A. S. Daniel, F. Meng, A. Forticaux, L. Li and S. Jin, J. Am. Chem. Soc., 2013, 135, 10274-10277.

D. Voiry, M. Salehi, R. Silva, T. Fujita, M. Chen, T. Asefa, V. B. Shenoy, G. Eda and M. Chhowalla, Nano Lett., 2013, 13, 6222-6227. C. A. Papageorgopoulos and W. Jaegermann, Surf. Sci., 1995, 338, 83-93.
Kalantar-Zadeh, ACS Nano, 2013, 7, 10083-10093. X. Fan, P. Xu, D. Zhou, Y. Sun, Y. C. Li, M. A. T. Nguyen, M. Terrones and T. E. Mallouk, Nano Lett., 2015, 15, 59565960.

X. Ma, P. Zhang, Z. Wang, R. Wu, T. Jiang, H. Wang, Y. Zhao, S. Wu, Y. Xie, Y. Zhan and T. Nan, Nanotechnology, 2016, 28, 084001.

T. Payam, W. Jieqiong, X. Hui, F. D. Joel, A. Mumtaz Murat, Z. Chuan, K. Kaifei, B. Brett, Z. Lijie, Z. Puqin, H. Shaoming, Y. Sen, V. B. Frank, C. John and Z. Hao, Mater. Res. Express, 2016, 3, 75009.

Z. He and W. Que, Appl. Mater. Today, 2016, 3, 23-56. L. Samad, S. M. Bladow, Q. Ding, J. Zhuo, R. M. Jacobberger, M. S. Arnold and S. Jin, ACS Nano, 2016, 10, 7039-7046.

X. Feng, Q. Tang, J. Zhou, J. Fang, P. Ding, L. Sun and L. Shi, Cryst. Res. Technol., 2013, 48, 363-368.

C. Perumal Veeramalai, F. Li, H. Xu, T. W. Kim and T. Guo, RSC Adv., 2015, 5, 57666-57670.

X. Feng, Q. Tang, J. Zhou, J. Fang, P. Ding, L. Sun and L. Shi, Cryst. Res. Technol., 2013, 48, 363-368.

G. Liu, A. W. Robertson, M. M.-J. J. Li, W. C. H. H. Kuo, M. T. Darby, M. H. Muhieddine, Y.-C. C. Lin, K. Suenaga, M. Stamatakis, J. H. Warner and S. C. E. Tsang, Nat. Chem., 2017, 9, 810-816.

R. G. Compton and C. E. Banks, Understanding voltammetry, World Scientific, 2011.

S. Park, Y. Shao, J. Liu and Y. Wang, Energy Environ. Sci., 2012, 5, 9331-9344.

Y. Li, H. Wang, L. Xie, Y. Liang, G. Hong and H. Dai, J. Am. Chem. Soc., 2011, 133, 7296-7299.

S. Trasatti, J. Electroanal. Chem., 1972, 39, 163-184. N. Cheng, S. Stambula, D. Wang, M. N. Banis, J. Liu, A. Riese, B. Xiao, R. Li, T. K. Sham, L. M. Liu, G. A. Botton and X. Sun, Nat. Commun., 2016, 7, 1-9. 